\title{
Assessing HPC Failure Detectors for MPI Jobs *
}

\author{
Kishor Kharbas ${ }^{1}$, Donghoon Kim ${ }^{1}$, Torsten Hoefler ${ }^{2}$ and Frank Mueller ${ }^{1}$ \\ ${ }^{1}$ North Carolina State University, Raleigh, NC 27695-7534, mueller@cs.ncsu.edu \\ ${ }^{2}$ Nat'l Center for Supercomputing Applications, Univ. of Illinois at Urbana-Champaign, Urbana, IL 61801
}

\begin{abstract}
Reliability is one of the challenges faced by exascale computing. Components are poised to fail during largescale executions given current mean time between failure $(M T B F)$ projections. To cope with failures, resilience methods have been proposed as explicit or transparent techniques. For the latter techniques, this paper studies the challenge of fault detection.

This work contributes a study on generic fault detection capabilities at the MPI level and beyond. The objective is to assess different detectors, which ultimately may or may not be implemented within the application's runtime layer. A first approach utilizes a periodic liveness check while a second method promotes sporadic checks upon communication activities. The contributions of this paper are two-fold: (a) We provide generic interposing of MPI applications for fault detection. (b) We experimentally compare periodic and sporadic methods for liveness checking. We show that the sporadic approach, even though it imposes lower bandwidth requirements and utilizes lower frequency checking, results in equal or worse application performance than a periodic liveness test for larger number of nodes. We further show that performing liveness checks in separation from MPI applications results in lower overhead than interpositioning, as demonstrated by our prototypes. Hence, we promote separate periodic fault detection as the superior approach for fault detection.
\end{abstract}

\section{Introduction}

The current road map to exascale computing faces a number of challenges, one of which is reliability. Given the number of computing cores, projected to be as large as a million, with ten of thousands of multi-socket nodes, components are poised to fail during the execution of large jobs due to a decreasing mean time between failures (MTBF) $[25,31]$. When faults become the norm rather than the exception, the underlying system needs to provide a resilience

\footnotetext{
*This work was supported in part by NSF grants 1058779, 0958311, 0937908 .
}

layer to tolerate faults. Proposed methods for resilience range from transparent techniques, such as checkpointing and computational redundancy, to explicit handling, such as in probabilistic or fault-aware computing. The latter approach requires significant algorithmic changes and is thus best suited for encapsulation into numerical libraries [15]. This paper focuses on the former techniques. It builds on recently developed techniques such as checkpointing (with restarts or rollbacks) or redundant computing in highperformance computing $[3,6,13,21,34]$ or API extensions for checkpointing $[15,24]$. A common challenge of transparent resiliency lies in the detection of faults, which is also a requirement for fault-awareness as proposed in MPI-3 [1]. Such detection is the focus of this paper.

Previous work suggested guidelines on the theoretical methodology for designing fault detection services. However, a practical implementation still poses a challenge in terms of completeness and accuracy because of the diversity of parallel system environments in terms of both hardware and software, which exposes a number of complications due to potentially unreliable failure detectors [11].

The fault model of this work is that components are subject to fail-stop behavior. In other words, components either work correctly or do not work at all. Transient or byzantine failures are not considered. A component is an entire compute node or a network connection / link between any two nodes. In such a framework, we base fault detection on timeout monitoring between endpoints. The focus of our work is to study the impact of timeout monitoring on application behavior such as to perturb application performance as little as possible.

Contributions: In this paper, we implement a fault detector (FD) to detect failures of an MPI application. The objective is to assess different detectors, which ultimately may or may not be implemented within the application's runtime layer. An FD can be included at various layers of the software stack. First, we choose the MPI communication layer to implement the FD. We detect MPI communication failures and, at the same time, also utilize the MPI layer as a means to implement detection. This approach has the 
advantage that it does not require any failure detection support from the underlying software/hardware platform. Second, we implement a separate FD as stand-alone processes across nodes.

In this framework, we observe the effect of a failure, such as lack of response for communication between any two nodes due to node or network failures. We do not perform root cause analysis, which is orthogonal to our work. We assume that the system model provides temporal guarantees on communication bounds (sometimes also combined with computation bounds) called "partially synchronous" [30]. The FD utilizes a time-out based detection scheme, namely, a ping-pong based implementation with the following properties:

- Transparency: The FD can be embedded in MPI applications without any additional modification or side-byside to MPI applications. For the former, the FD runs independently with a unique communicator different from an application program. When MPI applications call MPI_Init, the FD module is activated for each MPI task (on each node) as an independent thread through the MPI profiling interposing layer.

- Portability: MPI applications can be compiled without the FD. Applications only need to be re-linked with the profiling layer of MPI and the FT module. It is not necessary for MPI applications to change in their environment, design or source code. The FD works for arbitrary MPI implementations and has been tested with MPICH, Open MPI, and the LAM/MPI-family.

- Scalability: We compare two modes for our FD: it either sends a check message sporadically whenever the application has invoked a communication routine or it performs periodic checks at configurable intervals.

The rationale behind sporadic and periodic liveness probing is that the former can be designed as low-cost background control messages that are only triggered when an application is causally dependent on other nodes. The latter, in contrast, can be designed independently of any communication pattern but requires constant out-of-band checking but is agnostic of application communication behavior.

Experimental results show that the FD satisfies the above three properties. The results further indicate that the sporadic approach imposes lower bandwidth requirements of the network for control messages and results in a lower frequency of control messages per se. Nonetheless, the periodic FD configuration is shown to result in equal or better application performance overall compared to a sporadic liveness test for larger number of nodes, which is a nonobvious result and one of our contributions. We also observe that separation of the FD results in lower overheads as opposed to integration into MPI application runtime layer.
Our resulting implementation can easily be combined with reactive checkpoint/restart frameworks to trigger restarts after components have failed $[2,5-10,12-14,17,17-20,22,23$, $26,27,29,32-36]$.

\section{Design}

In principle, an FD can be designed using a variety of communication overlays to monitor liveness. A traditional heartbeat algorithm imposes high communication overhead in an all-to-all communication pattern with a message complexity $\Theta\left(n^{2}\right)$ and a time complexity of $\Omega(n)$. This overhead can be high, and a single node does not need to inquire about liveness of all other nodes in an MPI application.

A tree-based liveness check results in $\Theta(n)$ messages with a $\Omega(\log (n))$ time complexity where the root node has a collective view of liveness properties. However, mid-level failures of the tree easily result in graph partitioning so that entire subtrees may be considered dysfunctional due to the topological mapping of the overlay tree onto a physical network structure (e.g., a multi-dimensional torus).

We have designed two principle types of failure detection mechanisms. First, we support a sporadic (or on-demand) FD. Second, we have devised a periodic, ring-based FD. The periodic FD can be integrated into MPI applications or may function as a stand-alone liveness test separate from MPI applications. These approaches differ in their liveness probing periods and their network overlay structure.

On $n$ processes, a ring structure results in $\Theta(n)$ messages for liveness checking and imposes $\mathcal{O}(1)$ time (for parallel liveness checks with immediate neighbors on the ring) or up to $\mathcal{O}(n)$ time (for sequential checking that has to propagate around the ring), yet liveness properties are only known about immediate neighbors. For MPI applications, we argue that local knowledge is sufficient to trigger reactive fault tolerance at a higher level. Hence, rings are chosen as one of our designs for the FD due to their low complexity. Rings are utilized for periodic checks. They are also chosen for sporadic checks in the presence of collective communication.

A point-to-point (parallel) liveness check imposes $\mathcal{O}(1)$ message and time overhead, and lifeless properties are only known to immediate neighbors on the ring. We chose a point-to-point check for sporadic liveness monitoring. Here, the FD sends a control message only if an MPI application calls an MPI point-to-point communication routine and the result is not received within a timeout period. Hence, the control message overhead of this approach may be zero when responses to application messages are received prior to timeout expiration. In such a setting, the overhead is localized to a node and amounts to request queuing and request cancellation (in the best case). 


\subsection{Failure Detector Types}

We have designed two principle types of failure detection mechanisms. First, we support a sporadic (or on-demand) FD. Second, we have devised a periodic, ring-based FD. These approaches differ in their liveness probing periods and their network overlay structure. Our objective is to study overheads in the best case, i.e., in the absence of failures. Due to low jitter in dedicated HPC interconnects, it is sufficient to study constant-interval based probes rather than general-purpose networking techniques based on variable round-trip time monitoring.

\subsubsection{Periodic Ring-Based Failure Detection}

In this approach, starting from initialization of the MPI environment, we form a ring-based network overlay structure wherein the $i$-th node probes the $(i+1)$-th node in the ring (see Figure 1(b)). Thus, each node probes its neighbor in the ring irrespective of whether there is any active application communication between the two nodes or not. The probing is performed until the application terminates, and MPI tasks are subject to an implicit barrier in MPI_Finalize() to gracefully terminate probing.

For MPI applications, we argue that local knowledge is sufficient to trigger reactive fault tolerance at a higher level.

Repairing the ring topology in the presence of failures is a well-studied area beyond the scope of this paper.

\subsubsection{Sporadic/On-demand Failure Detection}

In this approach, a node $p$ probes a node $q$ only if $p$ and $q$ are engaged in an application-level point-to-point message exchange. If $p$ needs to wait beyond a timeout interval for the communication to $q$ to complete, a control message from $p$ to $q$ is issued (see Figure 1(a)). This happens when node $p$ makes a blocking MPI call, such as MPI_Recv() or MPI_Wait(). Similarly, if the application is engaged in collective communication, such as MPI_Bcast(), and the MPI call does not return within a timeout interval, a ring-based liveness check is triggered. If the liveness test is successful but the application-level MPI call has not been completer the liveness check is periodically repeated.

The control message overhead of this approach may $b$ zero when responses to application messages are receive prior to timeout expiration. In such a setting, the overhea is localized to a node and amounts to request queuing an request cancellation (in the best case).

\section{Implementation}

Our implementation assumes that there are reliable uf per bounds on processing speeds and message transmissio times. If a node fails to respond within a time-out interva the node is assumed to have failed under this model (failstop model). The implementation builds on this assumption when a node starts probing another node. Node pairs are determined by a causal dependency implied from the application communication pattern (for sporadic point-to-point communication) or through network overlays (for sporadic collectives and all periodic liveness checks). Probing is implemented via ping-pong messages monitoring round trip time (RTT) timeouts. Probing for failure detection can be parametrized as follows: (a) INTER-PROBE: This interval determines the frequency of probing, i.e., the time between successive probes by a node. Longer values may cause late detection of failure while shorter intervals allow for early detection but increase the overhead imposed by control messages. (b) TIME-OUT: This interval determines the granularity of failure detection but also impacts the correctness of the approach. If the interval is chosen too small, a slow response may lead to false positives (detection of failure even though the node is functional). Conversely, a large interval may delay detection of failures. Determination of a "good" timeout interval is non-trivial, even if we assume an upper bound on network and processing delay (see above).

We have used the MPI profiling layer to implement one version of the FD module. Wrappers have been written for MPI functions. These wrappers take appropriate FD actions before and after calling the PMPI versions of the corresponding communication function. When the application calls MPI_Init(), a duplicate communicator, DUP_MPI_COMM_WORLD, is created, which is subsequently used to send control messages for failure detection. The software stack of the FD in conjunction with an application is depicted in Figure 2. Application-level MPI calls (depicted as Fortran calls) trigger a language-neutral wrapper before calling the interposed MPI function. In the PMPI wrapper, the native MPI function is called (prefixed with PMPI_). The fault detector governs back-channel exchanges of control message over the duplicated communicator. Another version of the FD implements periodic checks as stand-alone processes separate from an MPI application.

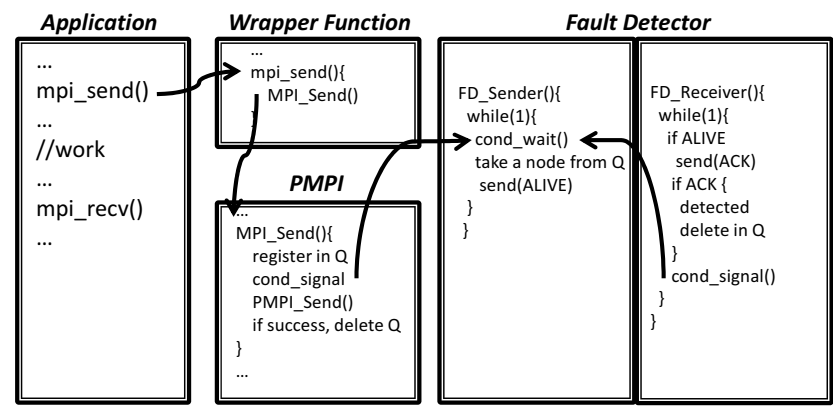

Figure 2. Application and FD Software Stacks 


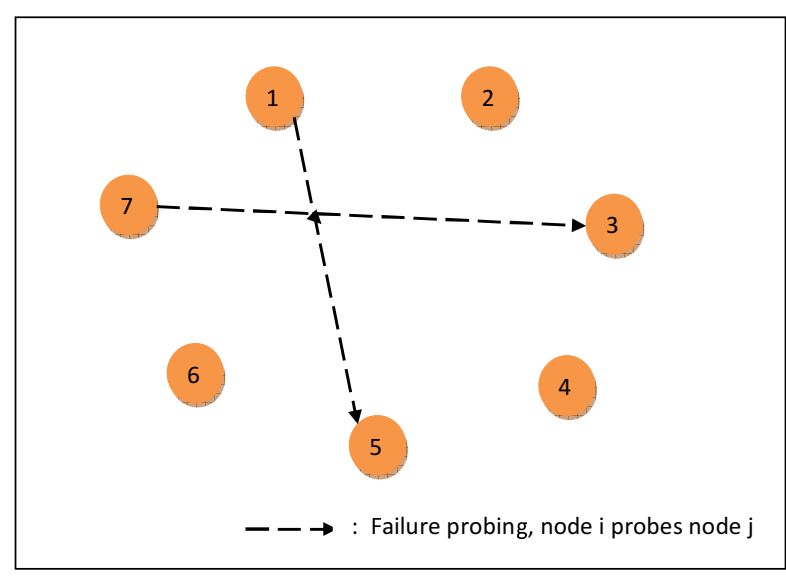

(a) Sporadic Fault Detection.

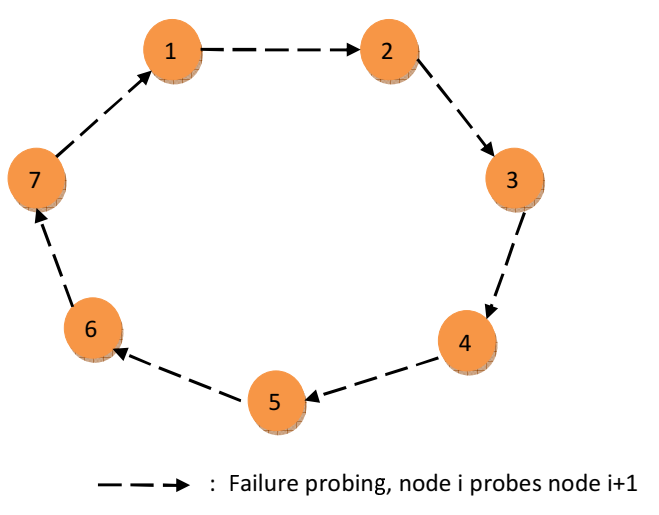

(b) Periodic Fault Detection.

Figure 1. Fault Detection Techniques

The fault detector is implemented as a pair of threads, namely sender and receiver threads. We require that MPI was initialized to support multi-threading, i.e., MPI_Init_thread() was called with, and returned MPI_THREAD_MULTIPLE to ensure thread support. The sender thread triggers an ALIVE message (ping) or waits for an acknowledgment (ACK) message (pong) up to a given timeout. The receiver thread receives ALIVE queries over the new communicator from the sender thread and responds with an ACK message. The failure detection module maintains a queue of events in sorted order of event times. An event could be "sending out a new probe to some node i" or "end of timeout interval for a probe sent to some node i". Upon such an event, the sender thread is activated and performs the respective action.

The sporadic and periodic failure detection mechanisms differ in the time when probing is started. The mechanisms also differ in terms of the communication pairs. In the periodic pattern (and collectives for the sporadic FD), source/sink of application-level MPI communication are ignored since back-channel communication is implemented over a ring overlay. Hence, requests are sent to a neighbor node $i+1$. For point-to-point sporadic FD, control messages follow the source/sink $(q / r)$ pairing that an application communication call utilizes. This difference is shown in Figure 3 . Notice that wildcard receives impose the same liveness check as collectives since endpoints are not known if a timeout is triggered before a wildcard receive is resolved. Figure 3 also illustrates that liveness checks are performed nonstop in the periodic case while they only occur in the presence of application communication for the sporadic case, even for collectives.

\section{Performance Evaluation}

We measured the overhead incurred by the FD module for the set of NAS parallel benchmarks (NPB V3.3) with

User Application Failure Detector

\section{Figure 3. Probes in Periodic and Sporadic Probing}

input classes C and D [4]. Using gettimeofday(), wall-clock times of applications were measured between MPI_Init() and MPI_Finalize() calls with and without failure detector interpositioning or backgrounding. Tests were performed by running each configuration five times and computing the average overhead for different inter-probe intervals and number of processes.

\subsection{Experimental Platform}

Experiments were conducted on a 108 node cluster with Infiniband QDR. Each node is a 2-way shared-memory multiprocessor with two octo-core AMD Opteron 6128 processors (16 cores per nodes). Each node runs CentOS 5.5 Linux x86_64. We used Open MPI 1.5.1 for evaluating the performance of the FD.

\subsection{Benchmark Results}

Figures 4 and 5 depict the relative overheads of fault detection for 128 processes (over 64 nodes) with periodic and sporadic fault detection, respectively. We omit our results for fewer processes as they show the same trends (with few exceptions, which are discussed). Overheads of the FD approach for fault tolerance with inter-probe frequencies of 


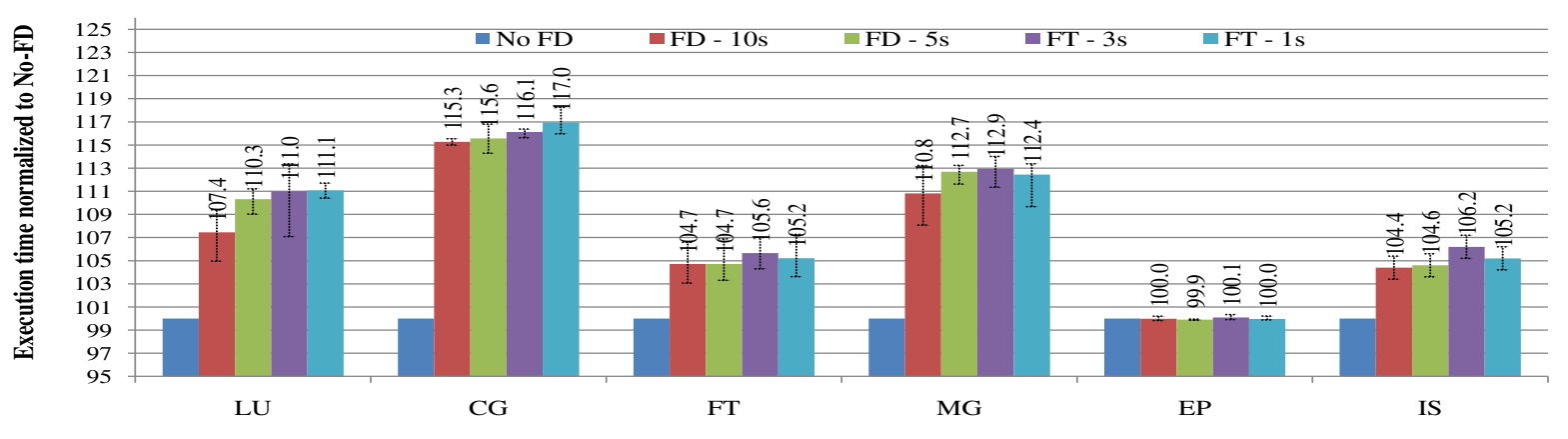

Figure 4. Overhead of Periodic Fault Detection for 128 Processes

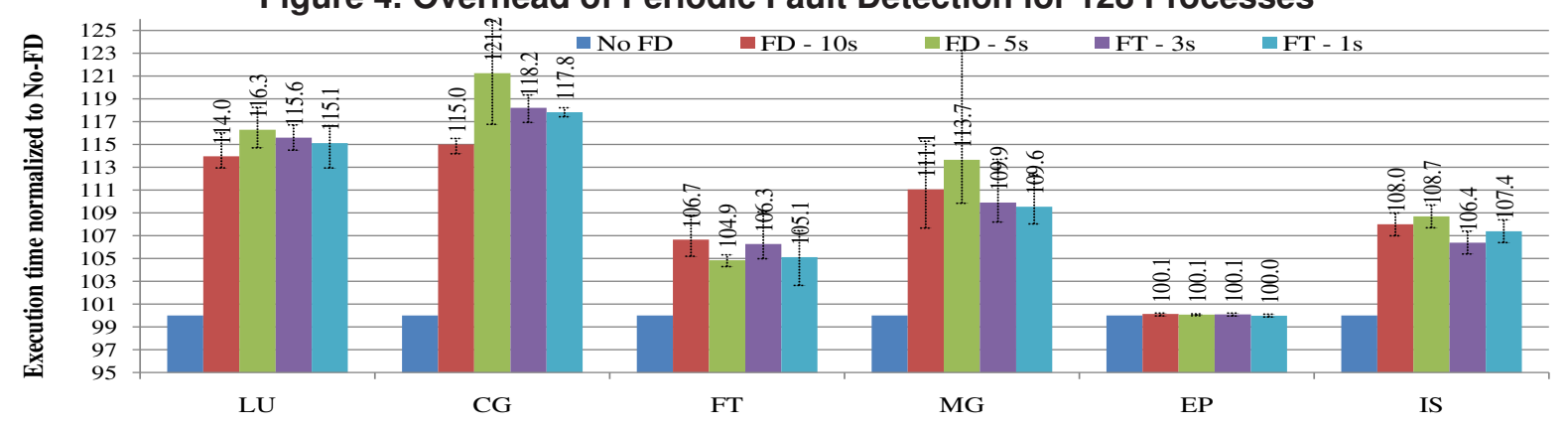

Figure 5. Overhead of Sporadic Fault Detection for 128 Processes

1-10 second ("FD 1sec" to "FD $10 \mathrm{sec")} \mathrm{are} \mathrm{plotted} \mathrm{relative}$ to application execution without fault tolerance ("No FD"), i.e., in the absence of the FD module (normalized to 100\%).

We first observe that both the sporadic and periodic FD have overheads ranging from less than $1 \%$ to $21 \%$ averaging around $10 \%$. We further observe that periodic either matches or outperforms by $2-6 \%$ the sporadic approach. This trend is also visible for smaller number of tasks (although less pronounced) and can be explained as follows: As overall communication is increasing, timeouts in the sporadic mode happen more frequently, in particular for collectives where communication results in contention (e.g., for all-to-all collectives). Sporadic control messages only add to this application-induced contention. In contrast, the periodic approach has the advantage that control messages are evenly likely to occur across the entire application duration. This probabilistic spread frequently results in control messages being issued during computation, i.e., when the network interconnect is not utilized at all. This trend increases with strong scaling (larger number of nodes).

We further conducted experiments with periodic liveness checking as a background activity in separate processes across nodes that an MPI application is running on. These experiments utilize a Gigabit Ethernet switch on the same cluster. The results depicted in Figure 6 show absolutely no overhead for NPB codes over 128 processes except for IS with an overhead of $4.5 \%$. Slight reductions in wall-clock time for CG and FT can be attributed to instruction cache benefits due to different code layout when the fault detector is linked with NPB codes. We also conducted experiments for background liveness checking using TCP over Infiniband resulting in only insignificantly lower execution time than these Ethernet experiments.

We also varied the number of MPI tasks per node and found these results to remain consistent up to 15 tasks per node. Only at 16 tasks per node did overheads spike to up to $28-60 \%$ depending on the NPB code with a high variance (see error bars) due to CPU-resource contention-both for Infiniband (Figure 7) and Ethernet (omitted). This shows that as long as a spare core is available for background activity, the impact of out-of-band communication on application performance is minimal. In HPC, applications tend to exploit the available per-node memory but may only utilize a subset of cores for high-end multi-core nodes due to limited memory bandwidth, which ensures that communication does not become a bottleneck [28].

We also investigated the impact of our FD approaches under Gigabit Ethernet embedded within the application as a sporadic and period approach (result omitted). We found that the performance of NPB codes is significantly higher for Ethernet as execution becomes constrained by network contention given the lower bandwidth available. Hence, the overhead of FD was overshadowed by contention of application messages and did not result in a noticeable overall impact. However, we consider such a high contention scenario not realistic for well-balanced, tuned HPC codes.

In addition to studying the overhead, we make the following observations based on application benchmark behavior from these results for sporadic liveness detection (Figure 5). 


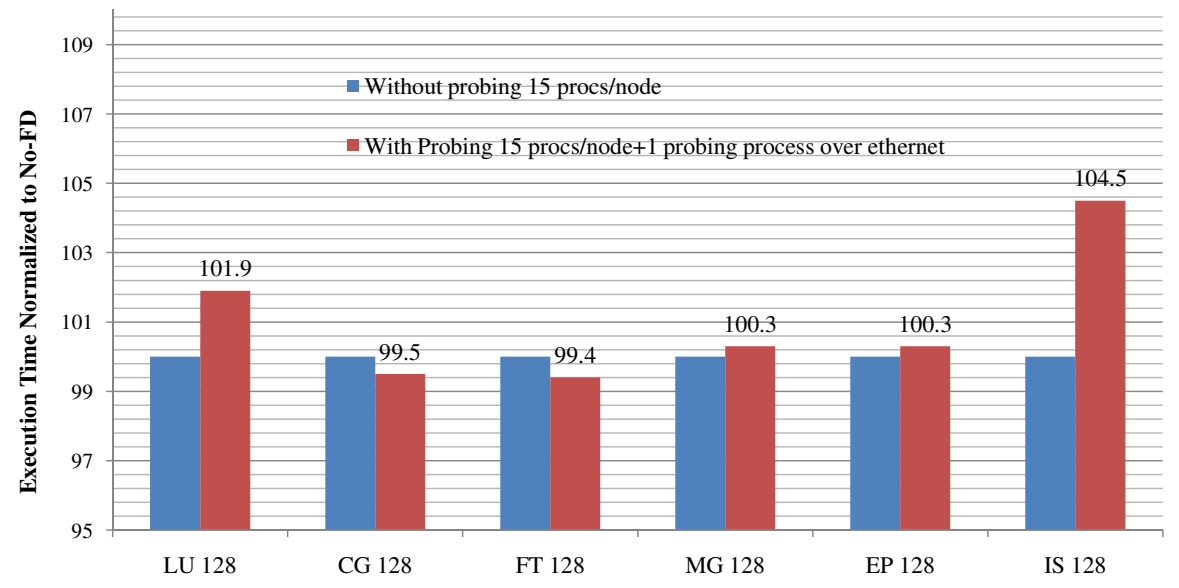

Figure 6. Overhead of Failure Detection as a Separate Process using Ethernet for 15 MPI tasks/node

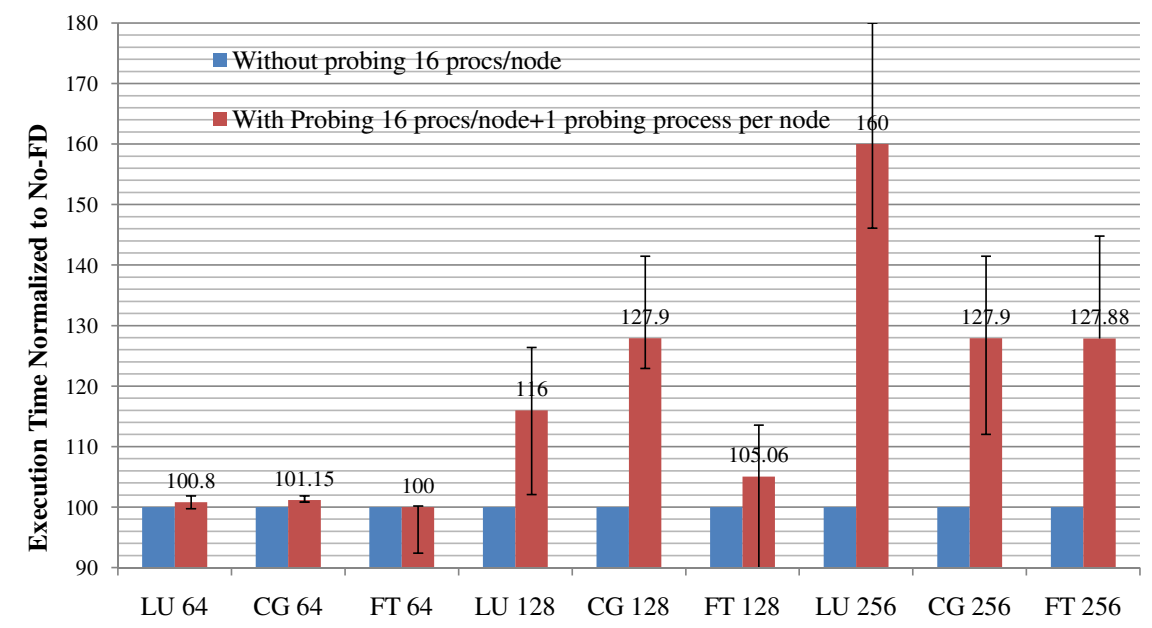

Figure 7. Overhead of Failure Detection as a Separate Process using Infiniband for 16 MPI tasks/node

The overhead of sporadic fault detection differs significantly for different benchmarks. Each MPI call from the application imposes modifications of the internal control message queues associated with the fault detection module, which adds some processing overhead. Hence, benchmarks with more frequent MPI calls suffer more overhead. For example, the CG, LU and MG benchmarks incur a larger number of total MPI calls and consequently incur higher overheads.

The value of the inter-probe interval does not have much significance in determining the overall overhead as most of the calls are completed within the sporadic inter-probe interval.

As the number of processes increases, so does the communication to computation ratio. Hence, the FD overhead increases as we increase the number of parallel processes. This effect can be seen more clearly in the LU and MG benchmarks where the average overhead of sporadic FD of 128 processes (Figure 5) is often larger than that for 64 and 32 processes.

Figure 4 shows the performance overhead of periodic liveness probing for 128 processes. We make the following observations based on application benchmark behavior from these results for periodic liveness detection.

The overhead of periodic fault detection also varies between different applications. Benchmarks with more frequent communication or larger messages suffer more overheads. For example, LU and CG are more communication intensive and thus cause more network contention between probe message traffic and application traffic, resulting in higher overhead. On the opposite side, benchmarks like EP, FT and IS are less communication intensive and result in less overhead. In most cases, the overhead of the FD approach increases when we decrease the inter-probe interval. 
As probing takes place from start to end, decrease in interprobe intervals generates a larger number of probes. This increases the processor utilization as well as network bandwidth demand, which results in increasing overheads. We conducted additional experiments with different numbers of processes and observed that the overhead of FD does not vary as we increase the scale of parallelism.

Overall, the results show that periodic failure detection performs better than sporadic for communication intensive codes and that separation of the FD from MPI applications reduces their perturbation.

\section{Related Work}

Chandra and Toueg classify eight classes of failure detectors by specifying completeness and accuracy properties [11]. They further show how to reduce the eight failure detectors to four and discuss how to solve the consensus problem for each class. This paper has influenced other contemporary work as it raises the problem of false positives for asynchronous systems. In our work, we focus on single-point failure detection. Consensus is an orthogonal problem, and we simply assume that a stabilization after multi-component failures eventually allows reactive fault tolerance, such as restarts from a checkpoint, to occur in a synchronous manner. Sastry et al. discuss the impact of celerating environments due to heterogeneous systems where absolute speeds (execution progress) could increase or decrease [30]. Bichronal timers with the composition of action clocks and real-time clocks are able to cope with celerating environments. Our work, in contrast, only relies on the clock of a local node. Genaud and Rottanapoka implemented a fault detector in a P2P-MPI environment utilizing a heartbeat approach [16]. They address failure information sharing, reason about a consensus phase and acknowledge overheads of fault detection due to their periodic heartbeat approach. Our work, in contrast, results in much lower message and time complexity. Consensus is orthogonal to our work, as discussed before.

\section{Conclusion}

In summary, our work contributes generic capabilities for fault detection/liveness monitoring of nodes and network links both at the MPI level and stand alone as prototypes. We designed and implemented two approaches to this end. The first approach utilizes a periodic liveness test and utilizes a ring-based network overlay for control messages. The second method promotes sporadic checks upon communication activities and relies on point-to-point control messages along the same communication paths utilized by the application, yet falls back to the ring-based overlay for collectives. We provide a generic interposing of MPI applications to realize fault detection for both cases plus a stand-alone version for the periodic case. Experimental results indicate that while the sporadic fault detector saves on network bandwidth by generating probes only when an MPI call is made, its messages are increasingly contending with application messages as the number of nodes increases. In contrast, periodic fault detection statistically avoids network contention as the number of processors increases. Overall, the results show that periodic failure detection performs better than sporadic for communication intensive codes and that separation of the FD from MPI applications reduces their perturbation. Production-style fault detections should thus become stand-alone programs (similar to our prototype) that can communicate back faults to the MPI runtime, which can then trigger reactive resilience mechanisms.

\section{References}

[1] Mpi-3 draft (with fault tolerance extensions). https://svn.mpi-forum.org/trac/mpi-forum-web/rawattachment/wiki/ft/run_through_stabilization/FTWGProcess-FT-Draft-6.pdf.

[2] S. Agarwal, R. Garg, M. S. Gupta, and J. E. Moreira. Adaptive incremental checkpointing for massively parallel systems. In ICS '04: Proceedings of the 18th annual international conference on Supercomputing, pages 277-286, New York, NY, USA, 2004. ACM.

[3] A. Agbaria and R. Friedman. Starfish: Fault-tolerant dynamic mpi programs on clusters of workstations. In Proc. of the 8th IEEE Intl. Symp. on High Perf. Distr. Comp., 1999.

[4] D. H. Bailey et al. The NAS Parallel Benchmarks. The International Journal of Supercomputer Applications, 5(3):63-73, Fall 1991.

[5] B. Barrett, J. M. Squyres, A. Lumsdaine, R. L. Graham, and G. Bosilca. Analysis of the component architecture overhead in Open MPI. In European PVM/MPI Users' Group Meeting, Sorrento, Italy, September 2005.

[6] G. Bosilca, A. Boutellier, and F. Cappello. MPICHV: Toward a scalable fault tolerant MPI for volatile nodes. In Supercomputing, Nov. 2002.

[7] B. Bouteiller, F. Cappello, T. Herault, K. Krawezik, P. Lemarinier, and M. Magniette. MPICH-V2: a fault tolerant MPI for volatile nodes based on pessimistic sender based message logging. In Supercomputing, 2003.

[8] S. Chakravorty, C. Mendes, and L. Kale. Proactive fault tolerance in large systems. In HPCRI: 1st Workshop on High Performance Computing Reliability Issues, in Proceedings of the 11th International Symposium on High Performance Computer Architecture (HPCA-11). IEEE Computer Society, 2005.

[9] S. Chakravorty, C. Mendes, and L. Kale. Proactive fault tolerance in MPI applications via task migra- 
tion. In International Conference on High Performance Computing, 2006.

[10] S. Chakravorty, C. Mendes, and L. Kale. A fault tolerance protocol with fast fault recovery. In Intl. Par. and Distrib. Proc. Symp., 2007.

[11] T. D. Chandra and S. Toueg. Unreliable failure detectors for reliable distributed systems. Journal of the ACM, 43:225-267, March 1996.

[12] F. Douglis and J. K. Ousterhout. Transparent process migration: Design alternatives and the sprite implementation. Softw., Pract. Exper., 21(8):757-785, 1991.

[13] J. Duell. The design and implementation of berkeley lab's linux checkpoint/restart. Tr, Lawrence Berkeley National Laboratory, 2000.

[14] E. N. Elnozahy and W. Zwaenepoel. Manetho: Transparent roll back-recovery with low overhead, limited rollback, and fast output commit. IEEE Trans. Comput., 41(5):526-531, 1992.

[15] G. E. Fagg and J. J. Dongarra. FT-MPI: Fault Tolerant MPI, supporting dynamic applications in a dynamic world. In Euro PVM/MPI Meeting, volume 1908, pages 346-353, 2000.

[16] S. Genaud and C. Rattanapoka. Evaluation of replication and fault detection in p2p-mpi. In Intl. Par. and Distrib. Proc. Symp., 2009.

[17] R. Gioiosa, J. C. Sancho, S. Jiang, and F. Petrini. Transparent, incremental checkpointing at kernel level: a foundation for fault tolerance for parallel computers. In Supercomputing, 2005.

[18] J. Heo, S. Yi, Y. Cho, J. Hong, and S. Y. Shin. Spaceefficient page-level incremental checkpointing. In SAC '05: Proceedings of the 2005 ACM symposium on Applied computing, pages 1558-1562, New York, NY, USA, 2005. ACM.

[19] S.-T. Hsu and R.-C. Chang. Continuous checkpointing: joining the checkpointing with virtual memory paging. Softw. Pract. Exper., 27(9):1103-1120, 1997.

[20] J. Hursey, J. M. Squyres, T. I. Mattox, and A. Lumsdaine. The design and implementation of checkpoint/restart process fault tolerance for Open MPI. In 12th IEEE Workshop on Dependable Parallel, Distributed and Network-Centric Systems, 032007.

[21] H. Jitsumoto, T. Endo, and S. Matsuoka. Abaris: An adaptable fault detection/recovery component framework for mpis. In Intl. Par. and Distrib. Proc. Symp., 2007.

[22] J. Mehnert-Spahn, E. Feller, and M. Schoettner. Incremental checkpointing for grids. In Linux Symposium, July 2009.

[23] D. S. Milojicic, F. Douglis, Y. Paindaveine, R. Wheeler, and S. Zhou. Process migration. ACM Computing Surveys (CSUR), 32(3):241-299, 2000.
[24] A. Moody, G. Bronevetsky, K. Mohror, and B. de Supinski. Design, modeling, and evaluation of a scalable multi-level checkpointing system. In Supercomputing, Nov. 2010.

[25] I. Philp. Software failures and the road to a petaflop machine. In HPCRI: 1st Workshop on High Performance Computing Reliability Issues. IEEE Computer Society, 2005.

[26] M. L. Powell and B. P. Miller. Process migration in DEMOS/MP. In Symposium on Operating Systems Principles, pages 110-119, Oct. 1983.

[27] J. Ruscio, M. Heffner, and S. Varadarajan. Dejavu: Transparent user-level checkpointing, migration, and recovery for distributed systems. In Intl. Par. and Distrib. Proc. Symp., 2007.

[28] J. Sancho, D. Kerbyson, and M. Lang. Characterizing the impact of using spare-cores on application performance. In Euro-Par Conference, pages 74-85, Sept. 2010.

[29] S. Sankaran, J. M. Squyres, B. Barrett, A. Lumsdaine, J. Duell, P. Hargrove, and E. Roman. The LAM/MPI checkpoint/restart framework: Systeminitiated checkpointing. In Proceedings, LACSI Symposium, Oct. 2003.

[30] S. Sastry, S. M. Pike, and J. L. Welch. Crash fault detection in celerating environments. In Intl. Par. and Distrib. Proc. Symp., 2009.

[31] B. Schroeder and G. Gibson. A large-scale study of failures in high-performance computing systems. In Proceedings of the 2006 International Conference on Dependable Systems and Networks (DSN-2006), Philadelphia, PA, June 2006.

[32] G. Stellner. CoCheck: checkpointing and process migration for MPI. In IEEE, editor, Proceedings of IPPS '96. The 10th International Parallel Processing Symposium: Honolulu, HI, USA, 15-19 April 1996, pages 526-531, 1109 Spring Street, Suite 300, Silver Spring, MD 20910, USA, 1996. IEEE Computer Society Press.

[33] J. Varma, C. Wang, F. Mueller, C. Engelmann, and S. L. Scott. Scalable, fault-tolerant membership for MPI tasks on hpc systems. In International Conference on Supercomputing, pages 219-228, June 2006.

[34] C. Wang, F. Mueller, C. Engelmann, and S. Scott. A job pause service under LAM/MPI+BLCR for transparent fault tolerance. In Intl. Par. and Distrib. Proc. Symp., Apr. 2007.

[35] C. Wang, F. Mueller, C. Engelmann, and S. Scott. Proactive process-level live migration in hpc environments. In Supercomputing, 2008.

[36] S. Yi, J. Heo, Y. Cho, and J. Hong. Adaptive pagelevel incremental checkpointing based on expected recovery time. In SAC '06: Proceedings of the 2006 ACM symposium on Applied computing, pages 1472 1476, New York, NY, USA, 2006. ACM. 